Article

\title{
Emergence of a Novel Dengue Virus 3 (DENV-3) Genotype-I Coincident with Increased DENV-3 Cases in Yangon, Myanmar between 2017 and 2019
}

\author{
Aung Min Soe ${ }^{1,2,3,4}{ }^{1}$, Mya Myat Ngwe Tun ${ }^{1}$, Takeshi Nabeshima ${ }^{1}$, Theingi Win Myat ${ }^{4}$, Moh Moh Htun ${ }^{4}$, \\ Htin Lin ${ }^{4}$, Nang Sarm Hom ${ }^{4}$, Shingo Inoue ${ }^{1,2}$, Khine Mya Nwe 1,2, Lynn Pa Pa Aye ${ }^{4}$, Mizuki Fukuta ${ }^{1,2}$, \\ Kyaw Zin Thant ${ }^{4}$, Futoshi Hasebe ${ }^{2,5}$, Kouichi Morita ${ }^{1,2}$, Sujan Shresta ${ }^{6, *(D)}$, Hlaing Myat Thu ${ }^{4, *}$ \\ and Meng Ling Moi ${ }^{1,2, *(D)}$
}

check for updates

Citation: Soe, A.M.; Ngwe Tun, M.M.; Nabeshima, T.; Myat, T.W.; Htun, M.M.; Lin, H.; Hom, N.S.; Inoue, S.; Nwe, K.M.; Aye, L.P.P.; et al. Emergence of a Novel Dengue Virus 3 (DENV-3) Genotype-I Coincident with Increased DENV-3 Cases in Yangon, Myanmar between 2017 and 2019. Viruses 2021, 13, 1152. https:// doi.org/10.3390/v13061152

Academic Editors: Luciana Barros de Arruda, Fabrício S. Campos and Flavio Guimaraes da Fonseca

Received: 30 April 2021

Accepted: 11 June 2021

Published: 16 June 2021

Publisher's Note: MDPI stays neutral with regard to jurisdictional claims in published maps and institutional affiliations.

Copyright: (C) 2021 by the authors Licensee MDPI, Basel, Switzerland. This article is an open access article distributed under the terms and conditions of the Creative Commons Attribution (CC BY) license (https:// creativecommons.org/licenses/by/ $4.0 /)$.
1 Department of Virology, Institute of Tropical Medicine, Nagasaki University, Nagasaki 852-8523, Japan; dr.aungminnsoe@gmail.com (A.M.S.); myamyat@tm.nagasaki-u.ac.jp (M.M.N.T.); mtmikami@tm.nagasaki-u.ac.jp (T.N.); pampanga@nagasaki-u.ac.jp (S.I.); drkhinemyanwe@gmail.com (K.M.N.); mixgoldenretriever@gmail.com (M.F.); moritak@nagasaki-u.ac.jp (K.M.)

2 Graduate School of Biomedical Sciences, Nagasaki University, Nagasaki 852-8523, Japan; rainbow@nagasaki-u.ac.jp

3 Program for Nurturing Global Leaders in Tropical and Emerging Communicable Diseases, Nagasaki University, Nagasaki 852-8501, Japan

4 Department of Medical Research, Yangon 11191, Myanmar; drtheingiwinmyat@gmail.com (T.W.M.); mohmoh.htun@gmail.com (M.M.H.); drhtinlin@gmail.com (H.L.); sarmhom@gmail.com (N.S.H.); dr.lynnpapaaye@gmail.com (L.P.P.A.); drkz.thant@gmail.com (K.Z.T.)

5 Research Center for Infectious Disease Research in Asia and Africa, Institute of Tropical Medicine, Nagasaki University, Nagasaki 852-8523, Japan

6 La Jolla Institute for Immunology, La Jolla, CA 92037, USA

* Correspondence: sujan@lji.org (S.S.); hmyatthu28@gmail.com (H.M.T.); sherry@nagasaki-u.ac.jp (M.L.M.); Tel.: +81-95-819-7829 (M.L.M.)

Abstract: Dengue fever, caused by the mosquito-borne dengue virus (DENV), has been endemic in Myanmar since 1970 and it has become a significant public health burden. It is crucial that circulating DENV strains are identified and monitored, and that their transmission efficiency and association with disease severity is understood. In this study, we analyzed DENV-1, DENV-2, DENV-3, and DENV-4 serotypes in 1235 serum samples collected in Myanmar between 2017 and 2019. Wholegenome sequencing of DENV-1-4 demonstrated that most DENV-1-4 strains had been circulating in Myanmar for several years. We also identified the emergence of DENV-3 genotype-I in 2017 samples, which persisted through 2018 and 2019. The emergence of the strain coincided with a period of increased DENV-3 cases and marked changes in the serotype dynamics. Nevertheless, we detected no significant differences between serum viral loads, disease severity, and infection status of individuals infected with different DENV serotypes during the 3-year study. Our results not only identify the spread of a new DENV-3 genotype into Yangon, Myanmar, but also support the importance of DENV evolution in changing the epidemic dynamics in endemic regions.

Keywords: dengue outbreak; Myanmar; co-circulation; serotypes; genotype-1; DENV-3

\section{Introduction}

Dengue virus (DENV) infection can be asymptomatic or cause an array of clinical symptoms that can include mild aches and pains, severe flu-like symptoms, and, less commonly, severe dengue composed of severe bleeding, organ impairment, plasma leakage/hypovolemic shock, and death [1-4]. In recent years, the incidence of DENV infection has increased in Myanmar, and the disease burden is now one of the largest among Southeast Asian countries. 
DENV is a member of the Flavivirus genus of the Flaviviridae family of single-stranded positive sense RNA viruses [5] and exists as four closely related serotypes, DENV-1-4 [6,7]. Although other regions in Southeast Asia have reported dengue outbreaks since the 1950s, Myanmar recorded its first outbreak in the 1970s. Since then, the prevalence of DENV infection has increased, particularly in recent years [8]. All four DENV serotypes have been detected in Myanmar, with DENV-1 was being the most dominant serotypes, while DENV-4 has been detected at a lesser frequency, since 2000s $[9,10]$. The largest DENV-1 epidemic in Myanmar to date occurred in 2015, when 42,913 cases and 140 deaths were recorded [11]. Recent studies have suggested that dengue epidemics are being shaped by the emergence of new DENV strains [12,13]. Thus, there is an urgent need to document and track the serotypes and genotypes of DENV circulating in Myanmar, not only to gain a better understanding of the epidemiological factors that drive DENV outbreaks, but also to facilitate the implementation of effective DENV control measures, including vaccination programs. Phylogenetic analyses have demonstrated the regular extinction of existing strains and emergence of new strains, the latter of which tends to coincide with increased viral transmission [14]. In turn, transmission may be further amplified by the introduction of new strains from other regions or the appearance of strains with higher transmission fitness resulting from selective pressures [14]. To better understand which DENV serotypes and genotypes have been circulating in Yangon, Myanmar in recent years, we performed full-genome next-generation sequencing and phylogenetic and nucleotide variation analyses of DENV-1-4 isolates collected in Myanmar between 2017 and 2019. We demonstrate changes in DENV dynamics in this endemic region and identify a newly emerged DENV-3 genotype-I (GI) that co-circulated with DENV-3 genotype-III (GIII) during this time.

\section{Materials and Methods}

\subsection{Patients and Sample Collection}

The study protocol was approved by the Institutional Review Boards of the two participating hospitals, which are Yangon General Hospital (patients $>12$ years old) and Yangon Children Hospital (patients $\leq 12$ years old). All patients or their legal guardians provided written informed consent. From 1235 individuals with suspected DENV infection, total of 1418 serum samples were collected (1235 acute serum samples and 183 convalescent serum samples) between June 2017 and December 2019. Suspected DENV infection was initially diagnosed based on clinical findings, and disease severity was classified according to the World Health Organization dengue diagnostic guideline (WHO, 2009). Initial DENV diagnostic testing was performed with SD BIOLINE Dengue NS1 Ag test kits (Standard Diagnostic Inc., Suwon, Korea). All serum samples were kept at $-80^{\circ} \mathrm{C}$ until analysis.

\subsection{Serological Confirmation of DENV Infection Status}

Serological confirmation of DENV infection and determination of infection status was performed using in-house anti DENV IgM-capture and anti-DENV IgG antibody enzyme-linked immunosorbent assays (ELISAs), and optical density (OD) values were read at $497 \mathrm{~nm}$ [15]. Sera were considered positive for IgM antibodies when the P/N ratios were $\geq 2$. To distinguish the infection status (primary and secondary dengue virus infections) in-house anti-DENV IgG ELISA system [15] was used. The test highly correlates with the dengue hemagglutination inhibition (DEN HI) test [16].

\subsection{Virus Isolation, RNA Extraction, and DENV Serotyping by RT-PCR}

Cultured Aedes albopictus mosquito clone cells (C6/36) were resuspended in Eagle's minimum essential medium supplemented with $2 \%$ fetal bovine serum (FBS) and $0.2 \mathrm{mM}$ non-essential amino acid. The cells were seeded in Nunclon Delta (flat) tubes (Thermo Fisher, Waltham, MA, USA), mixed with $10 \mu \mathrm{L}$ of patient serum and incubated at $28^{\circ} \mathrm{C}$ for 7 days. The culture supernatants were then harvested, transferred to fresh cultures of $\mathrm{C} 6 / 36$ cells, and the tubes were incubated for an additional 7 days [17]. After a total 
of 14 days, the cell culture supernatant was collected, and viral RNA was extracted and analyzed by reverse transcription-polymerase chain reaction (RT-PCR). Briefly, RNA was extracted using QIAamp RNA mini kits (Qiagen GmbH, Hilden, Germany) and amplified using Takara One-Step RT-PCR kits (Takara Bio Inc., Shiga, Japan) using consensus and DENV serotype-specific primers [18,19].

\subsection{Whole-Genome Sequencing}

First strand cDNA was synthesized from RNA that was isolated from the culture supernatants. Next, Superscript III and random primers (Thermo Fisher, Waltham, MA, USA) was used to generate the first strand CDNA, and the second strand cDNA was synthesized using NEBNext Second Strand Synthesis kits (New England Biolabs, Ipswich, MA, USA). DNA libraries were prepared using a Nextera XT DNA library prep kit (Illumina, San Diego, CA, USA) with aliquots of $1 \mathrm{ng}$ of DNA for tagmentation. Samples were quantified using a Qubit High Sensitivity dsDNA kit (Thermo Fisher, Waltham, MA, USA). Paired-end sequencing was performed using an Illumina MiSeq system with MiSeq sequencing reagent kits (v2, $2 \times 250$ bp; Illumina, San Diego, CA, USA).

\subsection{Phylogenetic Analysis and Amino Acid Variant Analysis}

The FASTX-Toolkit v0.0.14 was used for analysis. Adaptor sequences were trimmed, and quality was checked with FastQC v0.11.8 [20]. Trinity v2.8.4 was used for de novo assembly [21] and Blastn v2.7.1 [22] was used for contig assembly. Sequence identity was modified with SeqKit v10.0.1 [23] and fastq data was mapped with bwa v0.7.17 [24]. Amino acid variants were detected using Lofreq [25] and Varscan v2.4.3 [26], and the results were counter-checked with Pilon [27], V-phaser-2 [28], SNVer, and freebayes [29]. SAMtools v1.9 was then used to generate the consensus sequence [30]. Pre-processing of the data was performed using GATK v3.8.1 and Picard v2.20 [30]. Global sequences were obtained from the International Nucleotide Sequence Database Consortium and annotation was performed using SeqKit. Sequences were aligned using Mafft v7.407 [31]. The maximum likelihood phylogenetic tree was created with PhyML v3.2.0 [32] with 1000-replication bootstrap values, and substitution model (jModelTest v2.1.10) [33].

\subsection{Sanger Sequencing}

The E-gene region was also sequenced and confirmed by the Sanger method [10] using a Bigdye Dideoxy Terminator Sequencing kit v3.1 (Applied Biosystems, Foster, CA, USA), according to the manufacturer's instructions. Sequences were purified using Agencourt CleanSEQ kit (Agencourt Bioscience, Beverly, MA, USA) and analyzed using a 3730 DNA Analyzer (Applied Biosystems, Foster, CA, USA) [34].

\subsection{Quantification of DENV in Serum Samples}

Viremia levels in serum samples were quantified using two assays: an infectious virus plaque assay with the Fc $\gamma$ R-expressing BHK cell line [35] and a quantitative (q) RT-PCR assay.

For the plaque assay, BHK cells were resuspended in Eagle's Minimum Essential Medium (Sigma-Aldrich, Gillingham, UK) supplemented with 10\% FCS and $0.5 \mathrm{mg} / \mathrm{mL}$ neomycin (G418) (PAA Laboratories $\mathrm{GmbH}$, Pasching, Austria) and grown to confluence. The serum samples were serially diluted 10-fold (1:100-1:10,000) with EMEM, an aliquot of $200 \mu \mathrm{L}$ of each dilution was added to the confluent BHK cells, and the plates were incubated at $37^{\circ} \mathrm{C}$ for $1 \mathrm{~h}$. The cells were then overlaid with $1.5 \mathrm{~mL}$ of maintenance medium and the plates were incubated at $37^{\circ} \mathrm{C}$ with $5 \% \mathrm{CO}_{2}$ for 5 days. The cells were then fixed with $4 \%$ paraformaldehyde, stained with $0.25 \%$ crystal violet, and visualized under an inverted microscope. Plaques were counted and viral levels are expressed as plaque forming units (PFU) per $\mathrm{mL}$ of serum.

For qRT-PCR analysis, $5 \mu \mathrm{L}$ of RNA (extracted from the serum samples directly) was amplified the envelope gene by TaqMan real time RT-PCR system (Life Technologies, 
Carlsbad, CA, USA) [30,36]. Virus genome levels were expressed as log10 genome copies per $\mathrm{mL}$ of serum.

\subsection{Statistical Analyses}

Data were analyzed using Prism v9.0.2 software (GraphPad, San Diego, CA, USA) and compared using one way analysis of variance (ANOVA) and Student's $t$-test. A $p$ value of $<0.05$ was considered statistically significant.

\section{Results}

\subsection{Clinicopathological Characteristics and Serostatus of Patients}

During the study period, 1235 acute serum samples were obtained; of these, 912 (73.8\%) samples were NS1 antigen positive (SD BIOLINE Dengue NS1 Ag test kits) between June 2017 and December 2019. To confirm positivity and assess infection status, these 912 samples were subjected to ELISA tests. In-house dengue IgM capture ELISA tests produced a positive result for $776(85.1 \%)$ of the 912 total cases. In-house dengue IgG indirect ELISA tests was used to identify $264(28.9 \%)$ cases as primary infection and, $648(71.1 \%)$ as secondary infection (Figure S1). During the study period, the number of hospitalized patients per year was highest in 2018, as indicated by analysis of the weekly hospitalization rate for 2017-2019 (Figure S2).

\subsection{Distribution of Circulating DENV Serotypes}

Among the 912 NS1-positive samples, a total of 212 isolates was obtained by using the $\mathrm{C} 6 / 36$ clone cells, and there was no mixed infection (Table 1 ). The majority of isolates collected in 2017 were DENV-4 $(n=20)$ and DENV-3 $(n=18)$ serotypes, whereas in both 2018 and 2019, DENV-3 ( $n=55$ and 25, respectively) and DENV-1 ( $n=30$ and 20, respectively) were the predominant serotypes. Over the entire 3-year period, the most prevalent serotype was DENV-3 $(n=98,46.2 \%)$, followed by DENV-1 $(n=64,30.1 \%)$, and DENV-4 $(n=42$, $19.8 \%$ ). Notably, DENV-2 was detected in only 8 of the 212 isolates during the study period.

Table 1. Distribution of DENV serotypes circulating in Yangon, Myanmar 2017-2019.

\begin{tabular}{ccccc}
\hline & \multicolumn{3}{c}{ Number of Isolates Per Year } \\
\hline Serotype & $\mathbf{2 0 1 7}$ & $\mathbf{2 0 1 8}$ & $\mathbf{2 0 1 9}$ & Total \\
\hline DENV-1 & 14 & 30 & 20 & 64 \\
DENV-2 & 0 & 3 & 5 & 8 \\
DENV-3 & 18 & 55 & 25 & 98 \\
DENV-4 & 20 & 18 & 4 & 42 \\
\hline & 52 & 106 & 54 & 212 \\
\hline
\end{tabular}

The majority of the patients that was positive by virus isolation were children $\leq 12$ years of age (160 out of $212,75.5 \%)$ (Table 2$)$. The mean age was $9.7( \pm 6.3)$ years and consisted of $120(56.7 \%)$ male and $92(43.3 \%)$ female participants. According to the WHO classification for dengue symptoms, $74(34.9 \%), 119(56.1 \%)$, and $19(9 \%)$ patients were classified as having dengue without warning signs (DWoWS), dengue with warning signs (DWWS) and severe dengue (SD), respectively. 
Table 2. Clinicopathological characteristics and laboratory findings of patients in the study cohort ${ }^{1}$.

\begin{tabular}{|c|c|c|c|c|c|c|}
\hline \multirow[b]{2}{*}{ Patient Characteristic } & \multicolumn{4}{|c|}{ Patients Positive for Each DENV Serotype (N, \%) } & \multirow[b]{2}{*}{$\begin{array}{c}\text { Total } \\
(n=212,100 \%)\end{array}$} & \multirow[b]{2}{*}{$p$ Value } \\
\hline & $\begin{array}{c}\text { DENV-1 } \\
(n=64,30.1 \%)\end{array}$ & $\begin{array}{c}\text { DENV-2 } \\
(n=8,3.8 \%)\end{array}$ & $\begin{array}{c}\text { DENV-3 } \\
(n=98,46.2 \%)\end{array}$ & $\begin{array}{c}\text { DENV-4 } \\
(n=42,19.8 \%)\end{array}$ & & \\
\hline Age (years) & $8.8( \pm 5.1)^{2}$ & $8.0( \pm 4.6)$ & $9.6( \pm 5.4)$ & $11.7( \pm 5.4)$ & $9.7( \pm 6.3)$ & \\
\hline \multicolumn{7}{|l|}{ Age group (years) } \\
\hline$\leq 12$ & $52(32.5 \%)$ & $7(4.4 \%)$ & $75(46.8 \%)$ & $26(16.3 \%)$ & $160(100 \%)$ & 0.111 \\
\hline$>12$ & $12(23 \%)$ & $1(2 \%)$ & $23(44.2 \%)$ & $16(30.8)$ & $52(100 \%)$ & \\
\hline \multicolumn{7}{|l|}{ Gender } \\
\hline Male & $30(46.8 \%)$ & $6(75 \%)$ & $64(65.3 \%)$ & $20(47.6 \%)$ & $120(56.7 \%)$ & $\underline{0.047}$ \\
\hline Female & $34(53.2 \%)$ & $2(25 \%)$ & $34(34.7 \%)$ & $22(52.4 \%)$ & $92(43.3 \%)$ & \\
\hline \multicolumn{7}{|l|}{ Signs and Symptoms } \\
\hline Rash & 3 & 0 & 8 & 3 & 14 & 0.717 \\
\hline Hess test & 62 & 7 & 94 & 38 & 201 & $\underline{0.038}$ \\
\hline $\begin{array}{l}\text { Coffee ground } \\
\text { vomiting }\end{array}$ & 4 & 1 & 13 & 1 & 19 & 0.161 \\
\hline Muscle pain & 6 & 1 & 19 & 12 & 38 & 0.081 \\
\hline Joint pain & 6 & 1 & 17 & 10 & 34 & 0.241 \\
\hline Abdominal pain & 21 & 2 & 31 & 15 & 69 & 0.932 \\
\hline Hepatomegaly & 41 & 5 & 39 & 20 & 105 & $\underline{0.027}$ \\
\hline Splenomegaly & 0 & 0 & 1 & 1 & 2 & $\overline{0.632}$ \\
\hline Drowsiness & 17 & 2 & 31 & 8 & 58 & 0.493 \\
\hline $\begin{array}{l}\text { Thrombocytopenia. } \\
(<150,000 / \mu \text { L platelets })\end{array}$ & 26 & 2 & 32 & 20 & 80 & 0.308 \\
\hline Epistaxis & 11 & 2 & 19 & 5 & 37 & 0.689 \\
\hline Melena & 0 & 0 & 7 & 1 & 8 & 0.106 \\
\hline \multicolumn{7}{|l|}{ Symptoms 3} \\
\hline Without warning signs & $27(42.2 \%)$ & $2(25 \%)$ & $27(27.5 \%)$ & $18(42.8 \%)$ & $74(34.9 \%)$ & \\
\hline With warning signs & $32(50 \%)$ & $6(75 \%)$ & $62(63.3 \%)$ & $19(45.2 \%)$ & $119(56.1 \%)$ & 0.284 \\
\hline Severe dengue & $5(7.8 \%)$ & 0 & $9(9.2 \%)$ & $5(12 \%)$ & $19(9 \%)$ & \\
\hline \multicolumn{7}{|l|}{ Anti-DENV IgM 4} \\
\hline Negative & $24(37.5 \%)$ & $3(37.5 \%)$ & $43(43.8 \%)$ & $12(28.6 \%)$ & $82(38.7 \%)$ & 0.397 \\
\hline Positive & $40(62.5 \%)$ & $5(62.5 \%)$ & $55(56.2 \%)$ & $30(71.4 \%)$ & $130(61.3 \%)$ & \\
\hline \multicolumn{7}{|l|}{ Type of infection ${ }^{5}$} \\
\hline Primary & $31(48.4 \%)$ & $1(12.5 \%)$ & $50(51 \%)$ & $5(11.9 \%)$ & $87(41 \%)$ & $\leq 0.001$ \\
\hline Secondary & $33(51.6 \%)$ & $7(87.5 \%)$ & $48(49 \%)$ & $37(88.1 \%)$ & $125(59 \%)$ & \\
\hline
\end{tabular}

${ }^{1}$ Patients was confirmed positive by virus isolation, ${ }^{2}$ Mean \pm standard deviation, ${ }^{3}$ WHO classification system, ${ }^{4}$ as determined by capture ELISA, ${ }^{5}$ as determined by in-house dengue IgG indirect ELISA, underline indicates statistically significant values $(p$ value $<0.05)$.

\subsection{Whole-Genome Sequencing and Phylogenetic Analysis}

A total of 96 virus isolates obtained from a second cell culture passage were analyzed by NGS and amino acid variants. The isolates was selected based on positive isolation during the second passage by random sampling. All DENV-1 isolates (33 strains) belonged to GI and, possessed high homology to DENV-1 strains from regions neighboring Myanmar (Figure 1). DENV-1 GI was associated with two distinct lineages in Myanmar. The 7 DENV2 isolates belonged to Asian GI (Figure 2). Of the 36 DENV-3 isolates, 22 were of GI and were phylogenetically closely related to strains found in China, Singapore, Bangladesh, and Malaysia (Figure 3). Notably, DENV-3 GI was not detected in Myanmar prior to 2017. Phylogenetic analyses suggested that the novel DENV-3 GI was present in Myanmar by June 2017 and has remained in circulation since then. Although transmission of DENV-4 increased during 2017-2019, the circulating genotype (GI) remained unchanged from that detected in previous years (Figure 4). 


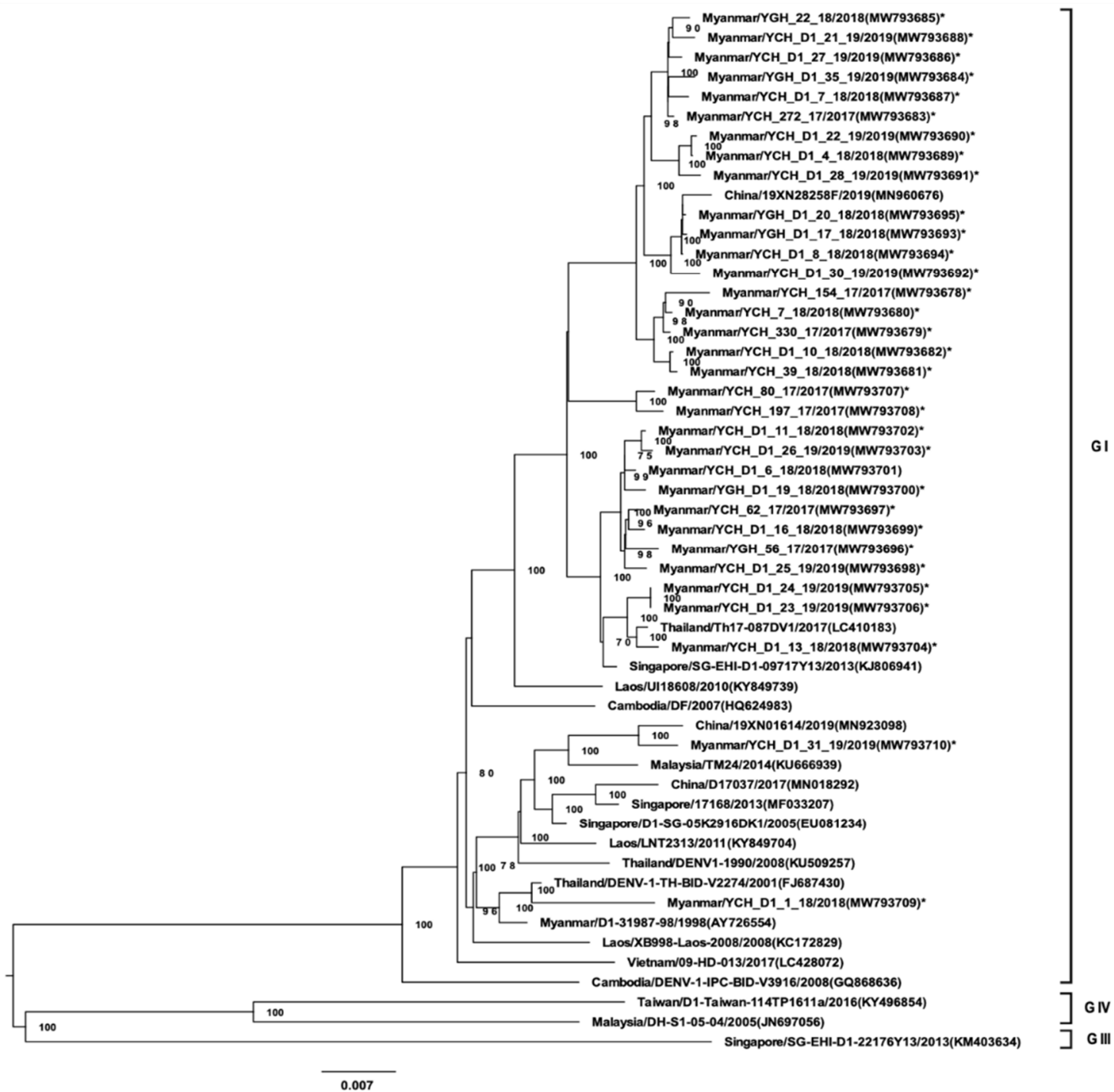

Figure 1. Phylogenetic tree of DENV-1 based on whole-genome sequencing. The maximum likelihood tree was generated using a general time reversible GTR $+\mathrm{I}+\mathrm{G}$ model with 1000 bootstrap replicates. Bootstrap values of $>70$ were indicated in the nodes. The tree shows global DENV strains, and the 33 DENV-1 isolates from this study (indicated by asterisks). These and representative strains of each genotype are named by country of origin, strain name, year of isolation, and accession number (GenBank). 


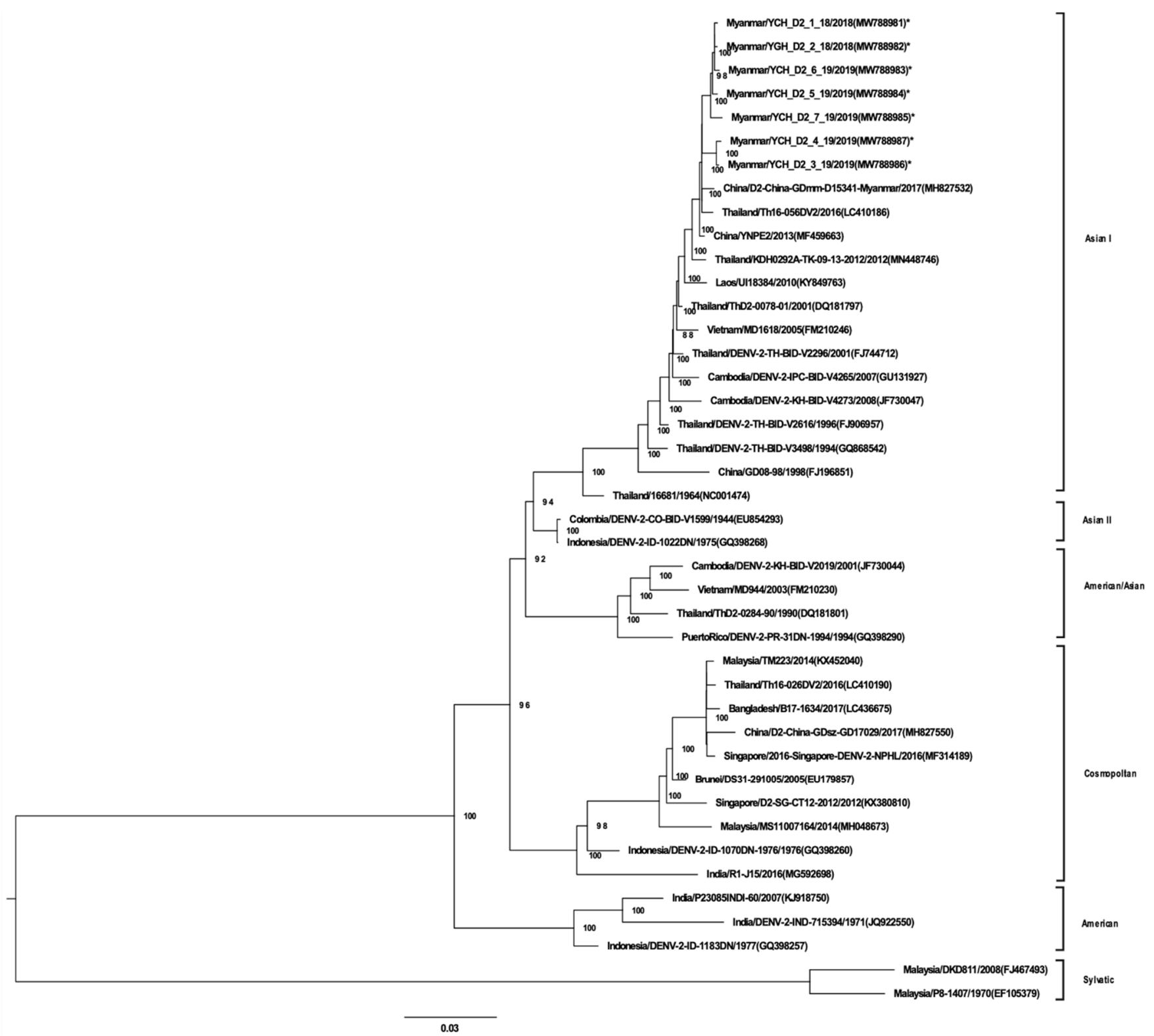

Figure 2. Phylogenetic tree of DENV-2 based on whole-genome sequencing. The maximum likelihood tree was generated using a general time reversible (GTR $+\mathrm{I}+\mathrm{G})$ model with 1000 bootstrap replicates. Bootstrap values of $>70$ were indicated in the nodes. The tree shows global DENV strains, and the 7 DENV-2 isolates from this study are indicated by asterisks. Representative strains of each genotype are named by country of origin, strain name, year of isolation, and accession number (GenBank). 


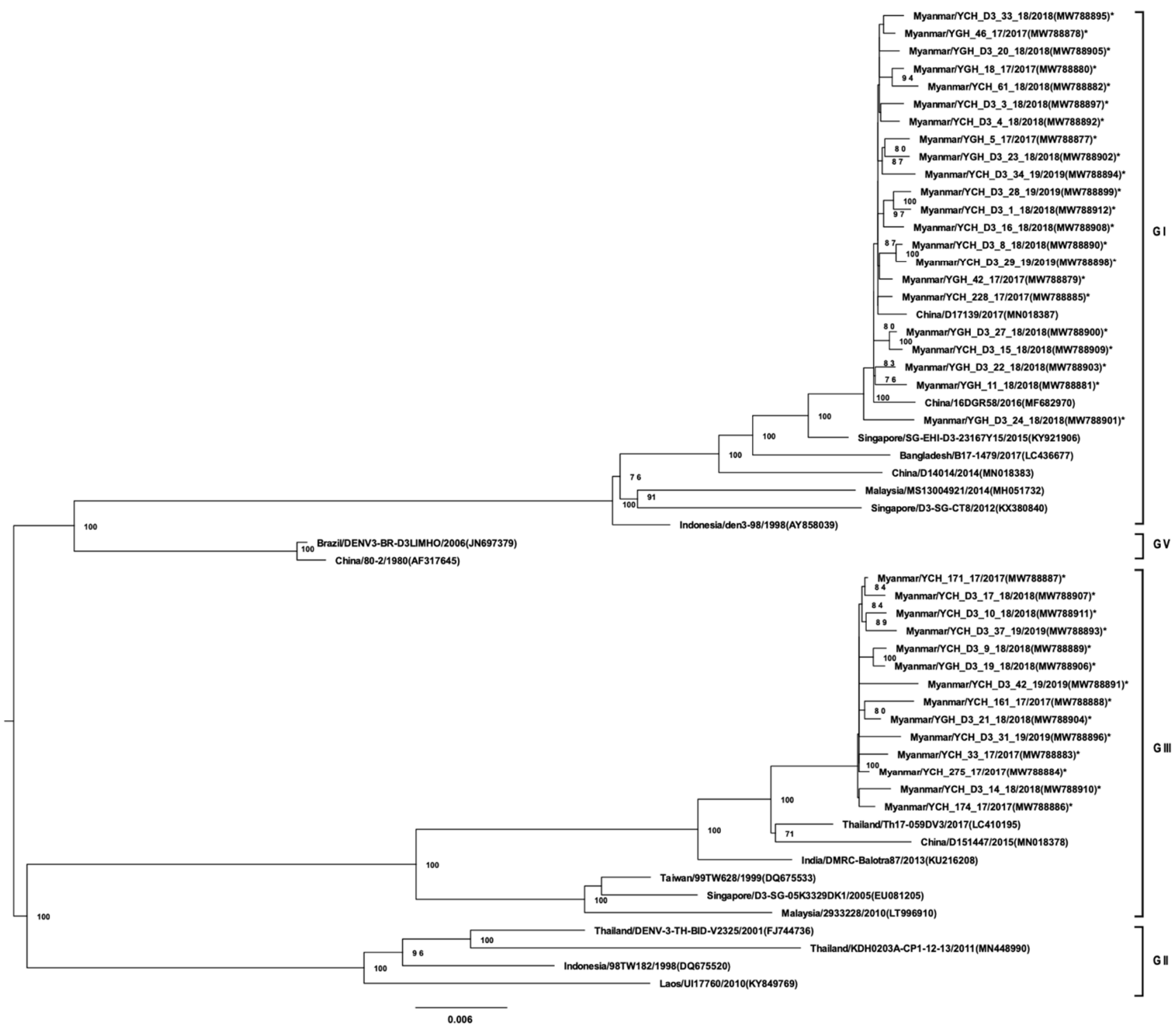

Figure 3. Phylogenetic tree of DENV-3 based on whole-genome sequencing. The maximum likelihood tree was generated using a general time reversible (GTR $+\mathrm{I}+\mathrm{G})$ model with 1000 bootstrap replicates. Bootstrap values of $>70$ were indicated in the nodes. The tree shows global DENV strains, and the 36 DENV-3 isolates from this study are indicated by asterisks. Representative strains of each genotype were named by country of origin, strain name, year of isolation, and accession number (GenBank). 


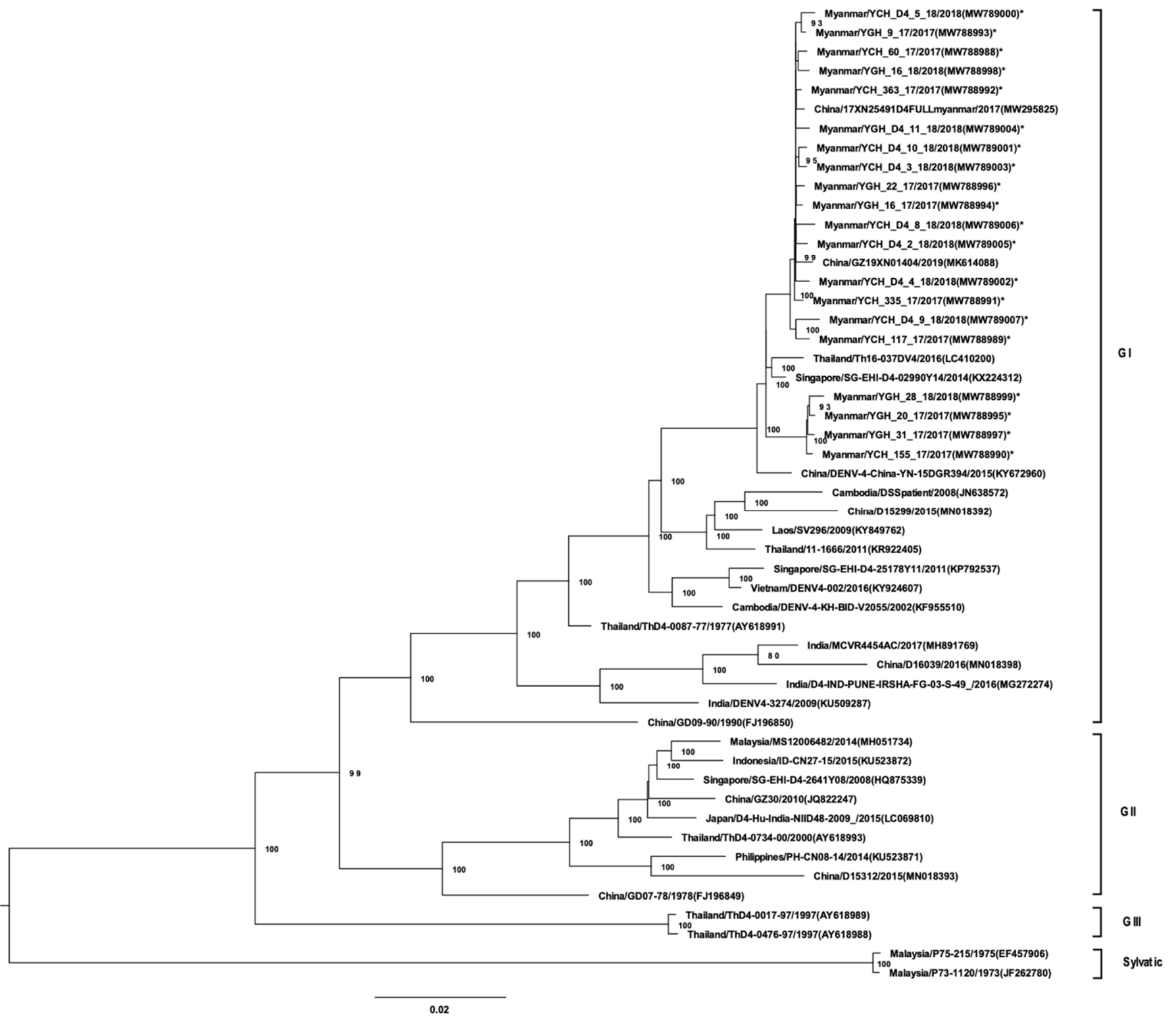

Figure 4. Phylogenetic tree of DENV-4 based on whole-genome sequencing. The maximum likelihood tree was generated using a general time reversible $(G T R+I+G)$ model with 1000 bootstrap replicates. Bootstrap values of $>70$ were indicated in the nodes. The tree shows global DENV strains, and the 20 DENV-4 isolates from this study are indicated by asterisks. Representative strains of each genotype were named by country of origin, strain name, year of isolation, and accession number (GenBank).

To elucidate the distribution patterns of the two genotypes of DENV-3 across the study period, partial E gene sequencing analyses of DENV-3 strains were performed by using Sanger sequencing (62 strains) and NGS (36 strains) (Figure 5). Overall, the results indicate that the DENV-3 genotype 1 had emerged in the country by June 2017, and it was maintained throughout the study period. In 2018 and 2019, nearly equal distribution of genotypes- 1 and -3 was observed. 


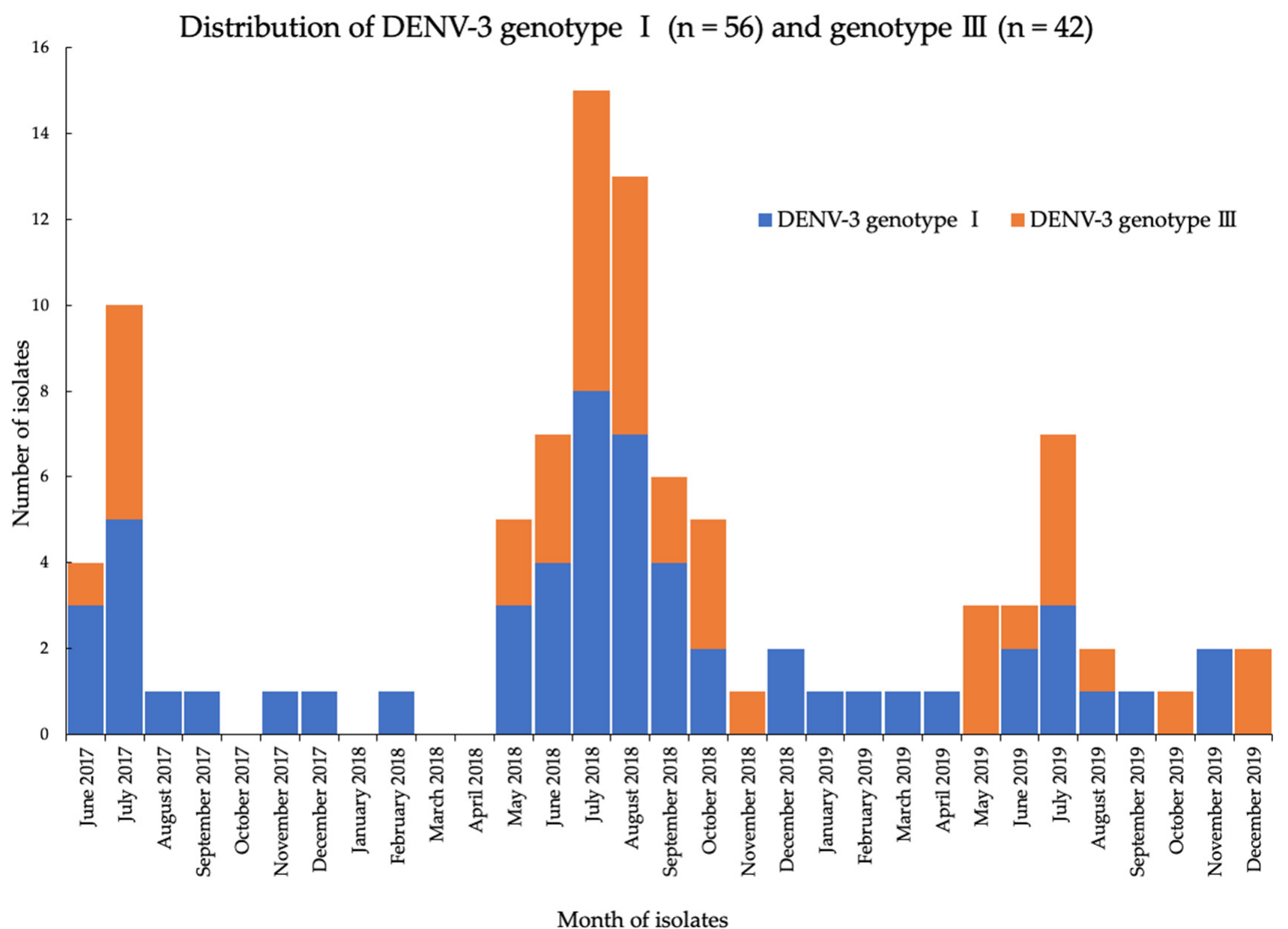

Figure 5. Distribution patterns of DENV-3 genotypes-I (blue) and -III (orange) co-circulating in Yangon, Myanmar during 2017-2019.

\subsection{DENV Amino Acid Variants}

We also analyzed amino acid variants in the DENV-1-4 isolates collected (Figure 6). Non-synonymous (NSY) and synonymous (SY) amino acid variants were identified by comparison to the reference strains DENV-1 AY726554, DENV-2 MF459663, DENV-3 KY921906, and DENV-4 KY672960.

Overall, amino acid variants of each serotype were predominantly due to NSY changes in all proteins, with the exception of DENV-3, which consisted of more SY variants than NSY variants (Figure 6). The majority of variants for all four DENV serotypes demonstrated NSY alterations in the NS5 protein, followed by NSY changes in the NS3 or E proteins. 

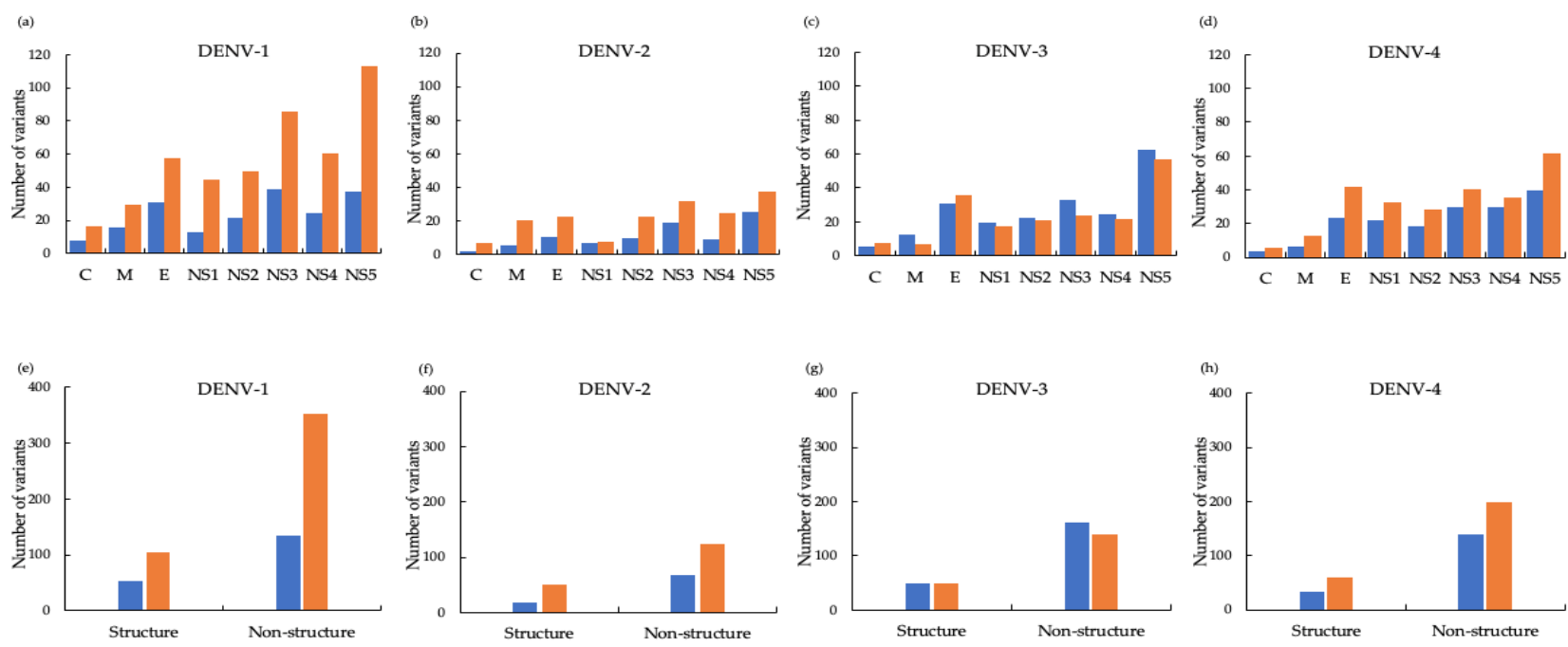

Figure 6. Amino acid variants of DENV-1-4 co-circulating in Yangon, Myanmar between 2017 and 2019. (a-d) Number of variants harboring synonymous (blue) and non-synonymous (orange) amino acid changes in the capsid (C), membraneassociated (M), envelope (E), and non-structural (NS1-5) proteins of (a) DENV-1, (b) DENV-2, (c) DENV-3, and (d) DENV-4. (e-h) Total number of variants with amino acid changes in structural (C,M,E) and non-structural (NS1-5) proteins. In total, 452 and 184, 169 and 82, 185 and 206, and 254 and 168 non-synonymous (orange) and synonymous (blue) variants, respectively, were identified for DENV-1, DENV-2, DENV-3, and DENV-4, respectively. For DENV-1, the number of NSY and SY variants in non-structural proteins was 350 and 132, respectively, compared with 102 and 52, respectively, in the structural proteins. The three most frequently mutated proteins for DENV-1 were NS5 (NSY 112, SY 37), followed by NS3 (NSY 85, SY 38), and E (NSY 57, SY30). Similarly, more DENV-2 variants harbored NSY than SY changes and more changes affected non-structural proteins (NSY 121 and SY 66, respectively) than structural proteins (NSY 48 and SY 16, respectively). The most frequently mutated DENV-2 proteins were NS5 (NSY 37, SY 25), NS3 (NSY 31, SY 18), and E (NSY 22, SY 10). For DENV-3, the total number of NSY and SY variants in non-structural proteins was 137 and 159, respectively, and in structural proteins was 48 and 47, respectively. The most frequently mutated DENV-3 proteins were NS5 (NSY 56, SY 62), E (NSY 35, SY 30), and NS3 (NSY 23, SY 32). Finally, for DENV-4, the total number of NSY and SY variants in non-structural proteins was 196 and 136, respectively, and in structural proteins was 58 and 32, respectively. The most frequently mutated were NS5 (NSY 61, SY 39), E (NSY 40, SY 23), and NS3 (NSY 40, SY 29).

\subsection{Associations between Viremia, Disease Severity, and Infection Status}

To determine whether DENV-1, -3 , and -4 infection loads correlated with disease severity in viremic patients, we quantified infectious virus by plaque assays and viral genomic copies by qRT-PCR (Figure 7). DENV-2 was not analyzed due to the small sample number $(n=8)$. Using the WHO classification system, we found no significant differences in viral levels between patients classified as having DWoWS, DWWS, or SD (Figure 7), although patients with SD showed a trend towards higher DENV-1 and DENV-4 burden measured by both assays. Similarly, although mean viral levels demonstrated a tendency to be higher in patients with primary infection than secondary infection, the differences were not statistically significant (Figure S3). 


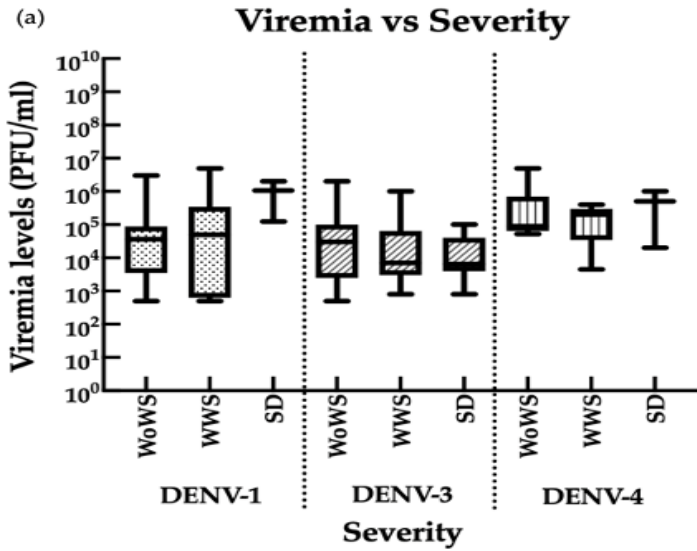

(c) Virus genome levels vs Severity

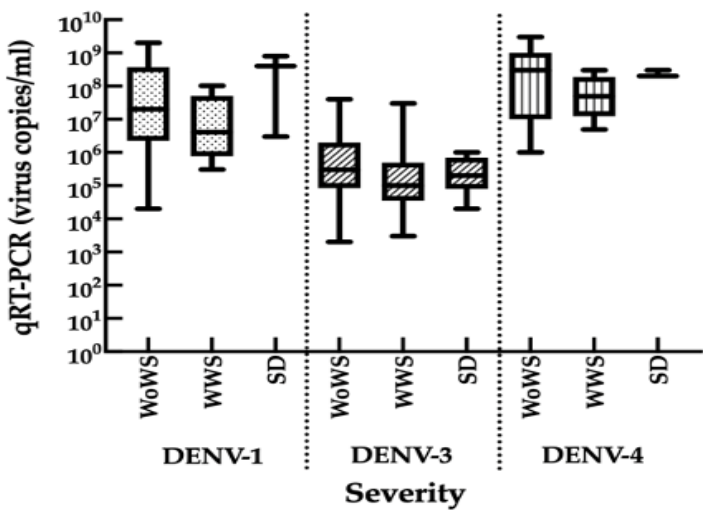

(b)

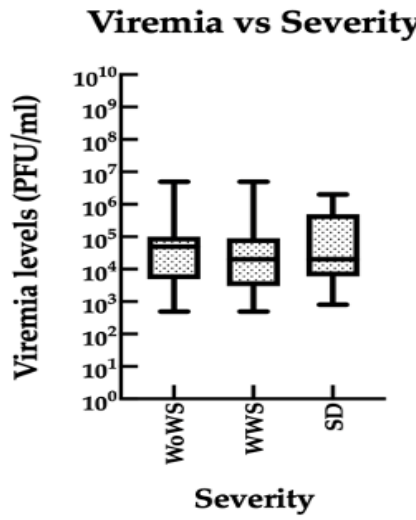

(d) Virus genome levels vs Severity

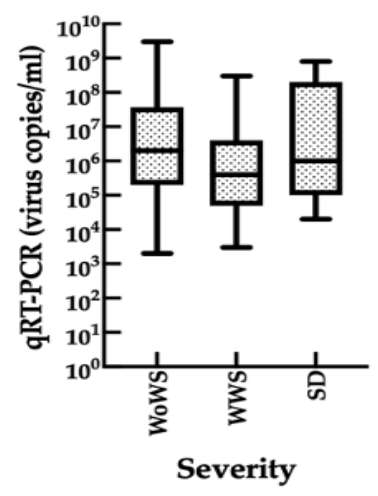

Figure 7. Comparison of viremia and disease severity in patients infected with DENV-1, DENV-3, and DENV-4. (a-d) Serum DENV-1 $(n=31)$, DENV-3 $(n=76)$, and DENV-4 $(n=15)$ levels determined by $(\mathbf{a}, \mathbf{b})$ plaque assay and $(\mathbf{c}, \mathbf{d})$ qRT-PCR. Data are presented for each serotype individually $(\mathbf{a}, \mathbf{c})$ or in combination $(\mathbf{b}, \mathbf{d})$. WoWS, without warning signs; WWS, with warning signs; SD, severe dengue (WHO classification). Boxplots show the median values (horizontal line in the box), $25-75 \%$ interquartile range (lower-upper limits of the box).

\section{Discussion}

Records of DENV serotypes and transmission in Myanmar before 2000 showed no distinct patterns; however, DENV-1 emerged as the dominant serotype in 2001 [9]. Between 2000 and 2017, DENV-1 was associated with several large disease outbreaks [9-11]. Although Myanmar and the surrounding region has been categorized as DENV endemic, relatively little is known about DENV outbreaks associated with serotypes other than DENV-1. Similarly, few studies have evaluated the evolutionary trajectory of DENV or the associations between serotype prevalence and clinical presentation. To address this gap, we performed a comprehensive analysis of the DENV serotypes and genotypes circulating in Myanmar between 2017 and 2019 and analyzed the association between the circulating DENV serotypes and clinical outcomes.

Phylogenetic analyses of DENV-1 performed since 2000, 2013, and 2015 have identified several major lineage turnovers is associated with DENV outbreaks and serotype dominance. In this study, we found that DENV-1, DENV-3, and DENV-4 serotypes were represented at approximately the same rates in 2017, despite the dominance of DENV-1 in earlier years. By 2018, the transmission dynamics in Myanmar had changed such that DENV-3 became the major circulating serotype in 2018 and 2019, followed by DENV-1. Thus, it is possible that serotype displacement and subsequent genotype shifting may be responsible for the sharp increase in DENV cases between 2017 and 2019, and may have contributed to the higher mortality rates (nearly 200 deaths) seen in 2017. 
In this study, we did not detect any changes in the genotypes of DENV-1, DENV-2, or DENV-4 circulating in Yangon, Myanmar, between 2017 and 2019. However, we identified a novel DENV-3 GI that emerged early in 2017 and persisted through the 3-year study period. This emergent DENV-3 GI was identified as the predominant genotype during several major dengue outbreaks in Southeast Asian countries, including Thailand. DENV3 GI was first detected in Thailand in 1988 [37], after which it increased, receded, and then reappeared during the 2015-2016 dengue season [38]. The strains identified here as circulating in Myanmar have high sequence homology with strains with high transmission activity circulating in neighboring countries (Indonesia, Malaysia, and Singapore) [39-42]. One of these countries is the most likely point of entry of the emergent strain into Myanmar, although the precise origin is unknown. The capital of Myanmar, Yangon, has a high population density and heavy human traffic due to socioeconomic factors. While DENV transmission in large urban areas has been suggested to be focal points for further spread to other regions, further studies are needed to determine whether the DENV transmission patterns in Yangon reflect those of other localities. While DENV-3 GI and GIII were cocirculating in Yangon, Myanmar, in 2017-2019, our results suggest that the emergent GI strain may have higher transmission activity, consistent with the increased infection rate in recent years.

Our analysis of DENV-1-4 amino acid variants revealed that NS5 was the most frequently mutated gene, followed by NS3 and E. The NS5 region encompasses the polymerase region that is highly conserved between serotypes and plays a key role in maintaining viral fitness and replication capacity [43]. Most of the variants of DENV-1, DENV-2, and DENV-4 were non-synonymous, while DENV-3 variants were predominantly due to synonymous changes in the NS5, NS3, and E proteins. This finding suggests that genotype expansion may have resulted from a combination of introduction of a new DENV-3 strain and its ongoing evolution at the local level. One factor proposed to be involved in driving of genotype/serotype turnover is genetic bottlenecks or positive selective pressures, which could confer an advantage for enhanced transmission that in turn leads to larger outbreaks.

Two hypotheses have been proposed to explain the marked differences in disease severity that can result from DENV infection: one is that viral mutation and evolution drive increased virulence [12] and the second is that pre-existing anti-DENV immunity may increase the severity of secondary infection through the process of antibody-enhancement of infection [13]. Although we found increases and decreases in the prevalence of DENV serotypes over the study period, we detected no significant differences between viremia, clinical severity and type of infection in patients infected with different serotypes. This result suggests that higher number of cases does not correlate with viral burden or clinical presentation; thus, further studies are warranted to determine whether an association exists between virus pathogenicity and transmission efficiency. Moreover, the observation that an increase in DENV-3 infections coincided with the emergence of a DENV-3 GI in Yangon, Myanmar suggests that the new strain may be more efficiently transmitted than the previously circulating strains. Further studies on viral dynamics and herd immunity will be important to understand the transmission dynamics of emergent strains in this DENV endemic country.

\section{Conclusions}

DENV infection is a major public health problem in Myanmar. We sequenced DENV serotypes circulating in Myanmar between 2017 and 2019 and compared them with historical data for DENV isolates spanning the preceding two decades. We identified a newly emerged DENV-3 GI that was introduced into Myanmar in 2017 and has persisted since then. Although a local DENV-3 GIII strain was also circulating in 2017-2019, the emergence of the DENV-3 GI coincided with a marked increase in DENV-3 transmission activity, suggesting that it may be the more pathological strain. Overall, our results suggest an important role for DENV genotype evolution in transmission dynamics in this endemic 
region, and additionally underscore the crucial need to monitor viral dynamics on an ongoing basis.

Supplementary Materials: The following materials are available online at https:/ /www.mdpi.com/ article/10.3390/v13061152/s1, Figure S1: Flow diagram of the methods used for this study, Figure S2: Number of admitted cases per week per year of NS1 positive samples $\{2017(n=282), 2018(n=440)$ and $2019(n=190)\}$ during the study period, Figure S3. Comparison of viremia and infection status in patients infected with DENV-1, DENV-3, and DENV-4.

Author Contributions: Conceptualization, M.L.M., H.M.T., and S.S.; Methodology, A.M.S., M.M.N.T., S.I., T.N., K.M., and M.L.M.; Sample Collection, T.W.M., M.M.H., H.L., N.S.H., L.P.P.A., and H.M.T.; Validation, A.M.S., M.M.N.T., and M.L.M.; Formal Analysis, A.M.S., S.I., T.N., M.M.N.T., and M.L.M.; Investigation, A.M.S., M.F., L.P.P.A., M.M.N.T., K.M.N., and M.L.M.; Resources, K.Z.T., F.H., K.M., M.M.N.T., and M.L.M.; Data Curation, A.M.S., M.M.N.T., and M.L.M.; Writing-Original Draft Preparation, A.M.S.; Writing—Review and Editing, A.M.S., M.M.N.T., K.Z.T., S.S., H.M.T., and M.L.M.; Visualization, A.M.S. and T.N.; Supervision, M.L.M.; Project Administration, H.M.T., S.S., and M.L.M.; Funding Acquisition, H.M.T., S.S., K.M., and M.L.M. All authors have read and agreed to the published version of the manuscript.

Funding: This research was supported by Japan Agency for Medical Research and Development (AMED) under e-ASIA Joint Research Program (19jm0210047h0004), AMED Japan Program for Infectious Diseases Research and Infrastructure (JP21wm0125006 and JP21wm0225018); Research on Emerging and Re-emerging Infectious Diseases (21fk0108109h0003, 21fk0108123h1102) and Nagasaki University.

Institutional Review Board Statement: Ethical approval for this study was obtained from the Ethics Review Committee on Medical Research including Human Subjects, Department of Medical Research, Myanmar (Ethics/DMR/2017/068), and from the Institute of Tropical Medicine, Nagasaki University, Japan (170707205).

Informed Consent Statement: Informed consent was obtained from adult patients and parents or legal guardians of children prior to sample collection.

Data Availability Statement: Dengue virus sequence data obtained from the samples in this study has been deposited in National Center for Biotechnology Information (NCBI) database (accessions number of each strain is as indicated in the virus strain name in Figures 1-4). The datasets generated during and/or analyzed during the current study are available from the corresponding authors on reasonable request.

Acknowledgments: The authors would like to thank the members of the Department of Virology, Institute of Tropical Medicine, Nagasaki University for providing technical advice and support. The authors are grateful to the healthcare staff for the support received during sample collection from hospitals and health institutions in Yangon, Myanmar. A.M.S. and K.M.N. are recipients of the Japanese Government (Monbukagakusho) Scholarship from the Ministry of Education, Science Sport and Culture of Japan under the eASIA Joint Research Program. A.M.S., K.M.N. and F.M. are grateful for academic support from the Program for Nurturing Global Leaders in Tropical and Emerging Communicable Diseases of the Graduate School of Biomedical Sciences, Nagasaki University.

Conflicts of Interest: The authors declare no conflict of interest. The funders had no role in the design of the study; in the collection, analyses, or interpretation of data; in the writing of the manuscript; or in the decision to publish the results.

\section{References}

1. About Dengue: What You Need to Know I Dengue / CDC. Available online: https://www.cdc.gov/dengue/about/index.html (accessed on 14 March 2021).

2. WHO/Dengue and Severe Dengue. Available online: https://apps.who.int/mediacentre/factsheets/fs117/en/index.html (accessed on 14 March 2021).

3. Bhatt, S.; Gething, P.W.; Brady, O.J.; Messina, J.P.; Farlow, A.W.; Moyes, C.L.; Drake, J.M.; Brownstein, J.S.; Hoen, A.G.; Sankoh, O.; et al. The Global Distribution and Burden of Dengue. Nature 2013, 496, 504-507. [CrossRef] [PubMed]

4. Dengue Fever-An Overview I ScienceDirect Topics. Available online: https:/ / www.sciencedirect.com/topics/immunologyand-microbiology / dengue-fever (accessed on 16 March 2021). 
5. Murugesan, A.; Manoharan, M. Dengue Virus. In Emerging and Reemerging Viral Pathogens: Volume 1: Fundamental and Basic Virology Aspects of Human, Animal and Plant Pathogens; Elsevier: London, UK, 2020; pp. 281-359. [CrossRef]

6. Diamond, M.S.; Pierson, T.C. Molecular Insight into Dengue Virus Pathogenesis and Its Implications for Disease Control HHS Public Access. Cell 2015, 162, 488-492. [CrossRef] [PubMed]

7. Kuhn, R.J.; Zhang, W.; Rossmann, M.G.; Pletnev, S.V.; Corver, J.; Lenches, E.; Jones, C.T.; Mukhopadhyay, S.; Chipman, P.R.; Strauss, E.G.; et al. Structure of Dengue Virus: Implications for Flavivirus Organization, Maturation, and Fusion. Cell 2002, 108, 717-725. [CrossRef]

8. Oo, P.M.; Wai, K.T.; Harries, A.D.; Shewade, H.D.; Oo, T.; Thi, A.; Lin, Z. The Burden of Dengue, Source Reduction Measures, and Serotype Patterns in Myanmar, 2011 to 2015-R2. Trop. Med. Health 2017, 45, 1-11. [CrossRef] [PubMed]

9. Hlaing Myat, T.; Lowry, K.; Thein Thein, M.; Than Nu, S.; Aye Maung, H.; Kyu Kyu, K.; Kyaw Zin, T.; Soe, T.; Aaskov, J. Myanmar Dengue Outbreak Associated with Displacement of Serotypes 2, 3, and 4 by Dengue 1. Emerg. Infect. Dis. 2004, 10, $593-597$.

10. Ngwe Tun, M.M.; Kyaw, A.K.; Makki, N.; Muthugala, R.; Nabeshima, T.; Inoue, S.; Hayasaka, D.; Moi, M.L.; Buerano, C.C.; Thwe, S.M.; et al. Characterization of the 2013 Dengue Epidemic in Myanmar with Dengue Virus 1 as the Dominant Serotype. Infect. Genet. Evol. 2016, 43, 31-37. [CrossRef] [PubMed]

11. Kyaw, A.K.; Ngwe Tun, M.M.; Moi, M.L.; Nabeshima, T.; Soe, K.T.; Thwe, S.M.; Myint, A.A.; Maung, K.T.T.; Aung, W.; Hayasaka, D.; et al. Clinical, Virological and Epidemiological Characterization of Dengue Outbreak in Myanmar, 2015. Epidemiol. Infect. 2017, 145, 1886-1897. [CrossRef]

12. Rico-Hesse, R. Microevolution and Virulence of Dengue Viruses. In Advances in Virus Research; Academic Press: San Diego, CA, USA, 2003; Volume 59, pp. 315-341. [CrossRef]

13. Halstead, S.B. The Alexander D. Langmuir Lecture the Pathogenesis of Dengue. Am. J. Epidemiol. 1981, 114, 632-648. [CrossRef]

14. Murray, N.E.A.; Quam, M.B.; Wilder-Smith, A. Epidemiology of Dengue: Past, Present and Future Prospects. Clin. Epidemiol. 2013, 5, 299-309. [CrossRef]

15. Inoue, S.; Alonzo, M.T.G.; Kurosawa, Y.; Mapua, C.A.; Reyes, J.D.; Dimaano, E.M.; Alera, M.T.P.; Saito, M.; Oishi, K.; Hasebe, F.; et al. Evaluation of a Dengue IgG Indirect Enzyme-Linked Immunosorbent Assay and a Japanese Encephalitis IgG Indirect Enzyme-Linked Immunosorbent Assay for Diagnosis of Secondary Dengue Virus Infection. Vector Borne Zoonotic Dis. 2010, 10, 143-150. [CrossRef]

16. Dengue: Guidelines for Diagnosis, Treatment, Prevention and Control—PubMed. Available online: https://pubmed.ncbi.nlm. nih.gov/23762963/ (accessed on 16 March 2021).

17. Ngwe Tun, M.M.; Thant, K.Z.; Inoue, S.; Nabeshima, T.; Aoki, K.; Kyaw, A.K.; Myint, T.; Tar, T.; Maung, K.T.T.; Hayasaka, D.; et al. Detection of East/Central/South African Genotype of Chikungunya Virus in Myanmar, 2010. Emerg. Infect. Dis. 2014, 20, 1378-1381. [CrossRef]

18. Lanciotti, R.S.; Calisher, C.H.; Gubler, D.J.; Chang, G.J.; Vorndam, A.V. Rapid Detection and Typing of Dengue Viruses from Clinical Samples by Using Reverse Transcriptase-Polymerase Chain Reaction. J. Clin. Microbiol. 1992, 30, 545-551. [CrossRef] [PubMed]

19. Morita, K.; Tanaka, M.; Igarashi, A. Rapid Identification of Dengue Virus Serotypes by Using Polymerase Chain Reaction. J. Clin. Microbiol. 1991, 29, 2107-2110. [CrossRef] [PubMed]

20. Sudigyo, D.; Rahmawati, G.; Setiasari, D.W.; Poluan, R.H.; Sesotyosari, S.L.; Wardana, T.; Herawati, C.; Heriyanto, D.S.; Indrasari, S.R.; Afiahayati; et al. Transcriptome Profile of next Generation Sequence Data Related to Inflammation on Nasopharyngeal Carcinoma Cases in Indonesia. Asian Pac. J. Cancer Prev. 2020, 21, 2763-2769. [CrossRef] [PubMed]

21. Grabherr, M.G.; Haas, B.J.; Yassour, M.; Levin, J.Z.; Thompson, D.A.; Amit, I.; Adiconis, X.; Fan, L.; Raychowdhury, R.; Zeng, Q.; et al. Full-Length Transcriptome Assembly from RNA-Seq Data without a Reference Genome. Nat. Biotechnol. 2011, $29,644-652$. [CrossRef] [PubMed]

22. Camacho, C.; Coulouris, G.; Avagyan, V.; Ma, N.; Papadopoulos, J.; Bealer, K.; Madden, T.L. BLAST+: Architecture and Applications. BMC Bioinform. 2009, 10, 1-9. [CrossRef]

23. Shen, W.; Le, S.; Li, Y.; Hu, F. SeqKit: A Cross-Platform and Ultrafast Toolkit for FASTA/Q File Manipulation. PLoS ONE 2016, 11, e0163962. [CrossRef]

24. Li, H.; Durbin, R. Fast and Accurate Long-Read Alignment with Burrows-Wheeler Transform. Bioinformatics 2010, 26, 589-595. [CrossRef]

25. Wilm, A.; Aw, P.P.K.; Bertrand, D.; Yeo, G.H.T.; Ong, S.H.; Wong, C.H.; Khor, C.C.; Petric, R.; Hibberd, M.L.; Nagarajan, N. LoFreq A Sequence-Quality Aware, Ultra-Sensitive Variant Caller for Uncovering Cell-Population Heterogeneity from High-Throughput Sequencing Datasets. Nucleic Acids Res. 2012, 40, 11189-11201. [CrossRef]

26. Koboldt, D.C.; Zhang, Q.; Larson, D.E.; Shen, D.; McLellan, M.D.; Lin, L.; Miller, C.A.; Mardis, E.R.; Ding, L.; Wilson, R.K. VarScan 2: Somatic Mutation and Copy Number Alteration Discovery in Cancer by Exome Sequencing. Genome Res. 2012, 22, 568-576. [CrossRef]

27. Walker, B.J.; Abeel, T.; Shea, T.; Priest, M.; Abouelliel, A.; Sakthikumar, S.; Cuomo, C.A.; Zeng, Q.; Wortman, J.; Young, S.K.; et al. Pilon: An Integrated Tool for Comprehensive Microbial Variant Detection and Genome Assembly Improvement. PLoS ONE 2014, 9, e112963. [CrossRef]

28. Yang, X.; Charlebois, P.; Macalalad, A.; Henn, M.R.; Zody, M.C. V-Phaser 2: Variant Inference for Viral Populations. BMC Genomics 2013, 14, 674. [CrossRef] 
29. Sandmann, S.; De Graaf, A.O.; Karimi, M.; Van Der Reijden, B.A.; Hellström-Lindberg, E.; Jansen, J.H.; Dugas, M. Evaluating Variant Calling Tools for Non-Matched Next-Generation Sequencing Data. Sci. Rep. 2017, 7, 1-12. [CrossRef]

30. Ngwe Tun, M.M.; Muthugala, R.; Nabeshima, T.; Soe, A.M.; Dumre, S.P.; Rajamanthri, L.; Jayawardana, D.; Attanayake, S.; Inoue, S.; Morita, K. Complete Genome Analysis and Characterization of Neurotropic Dengue Virus 2 Cosmopolitan Genotype Isolated from the Cerebrospinal Fluid of Encephalitis Patients. PLoS ONE 2020, 15, e0234508. [CrossRef]

31. Katoh, K.; Standley, D.M. MAFFT Multiple Sequence Alignment Software Version 7: Improvements in Performance and Usability. Mol. Biol. Evol. 2013, 30, 772-780. [CrossRef]

32. Guindon, S.; Dufayard, J.F.; Lefort, V.; Anisimova, M.; Hordijk, W.; Gascuel, O. New Algorithms and Methods to Estimate Maximum-Likelihood Phylogenies: Assessing the Performance of PhyML 3.0. Syst. Biol. 2010, 59, 307-321. [CrossRef] [PubMed]

33. Darriba, D.; Taboada, G.L.; Doallo, R.; Posada, D. JModelTest 2: More Models, New Heuristics and Parallel Computing. Nat. Methods 2012, 9, 772. [CrossRef]

34. Thant, K.Z.; Ngwe Tun, M.M.; del Carmen Parquet, M.; Inoue, S.; Lwin, Y.Y.; Lin, S.; Aye, K.T.; Khin, P.T.; Myint, T.; Htwe, K.; et al. Molecular Epidemiology of Dengue Viruses Co-Circulating in Upper Myanmar in 2006. Trop. Med. Health 2015, 43, 21-27. [CrossRef] [PubMed]

35. Moi, M.L.; Lim, C.K.; Kotaki, A.; Takasaki, T.; Kurane, I. Detection of Higher Levels of Dengue Viremia Using Fc $\gamma$ R-Expressing BHK-21 Cells than Fc $\gamma$ R-Negative Cells in Secondary Infection but Not in Primary Infection. J. Infect. Dis. 2011, 203, $1405-1414$. [CrossRef] [PubMed]

36. Ito, M.; Takasaki, T.; Yamada, K.I.; Nerome, R.; Tajima, S.; Kurane, I. Development and Evaluation of Fluorogenic TaqMan Reverse Transcriptase PCR Assays for Detection of Dengue Virus Types 1 to 4. J. Clin. Microbiol. 2004, 42, 5935-5937. [CrossRef] [PubMed]

37. Wittke, V.; Robb, T.E.; Thu, H.M.; Nisalak, A.; Nimmannitya, S.; Kalayanrooj, S.; Vaughn, D.W.; Endy, T.P.; Holmes, E.C.; Aaskov, J.G. Extinction and Rapid Emergence of Strains of Dengue 3 Virus during an Interepidemic Period. Virology 2002, 301, 148-156. [CrossRef] [PubMed]

38. Hamel, R.; Surasombatpattana, P.; Wichit, S.; Dauvé, A.; Donato, C.; Pompon, J.; Vijaykrishna, D.; Liegeois, F.; Vargas, R.M.; Luplertlop, N.; et al. Phylogenetic Analysis Revealed the Co-Circulation of Four Dengue Virus Serotypes in Southern Thailand. PLOS ONE 2019, 14, e0221179. [CrossRef] [PubMed]

39. Shu, P.Y.; Su, C.L.; Liao, T.L.; Yang, C.F.; Chang, S.F.; Lin, C.C.; Chang, M.C.; Hu, H.C.; Huang, J.H. Molecular Characterization of Dengue Viruses Imported into Taiwan during 2003-2007: Geographic Distribution and Genotype Shift. Am. J. Trop. Med. Hyg. 2009, 80, 1039-1046. [CrossRef]

40. Ong, S.H.; Yip, J.T.; Chen, Y.L.; Liu, W.; Harun, S.; Lystiyaningsih, E.; Heriyanto, B.; Beckett, C.G.; Mitchell, W.P.; Hibberd, M.L.; et al. Periodic Re-Emergence of Endemic Strains with Strong Epidemic Potential-A Proposed Explanation for the 2004 Indonesian Dengue Epidemic. Infect. Genet. Evol. 2008, 8, 191-204. [CrossRef]

41. Sasmono, R.T.; Wahid, I.; Trimarsanto, H.; Yohan, B.; Wahyuni, S.; Hertanto, M.; Yusuf, I.; Mubin, H.; Ganda, I.J.; Latief, R.; et al. Genomic Analysis and Growth Characteristic of Dengue Viruses from Makassar, Indonesia. Infect. Genet. Evol. 2015, 32, 165-177. [CrossRef]

42. Sucipto, T.H.; Kotaki, T.; Mulyatno, K.C.; Churrotin, S.; Labiqah, A.; Soegijanto, S.; Kameoka, M. Phylogenetic Analysis of Dengue Virus in Bangkalan, Madura Island, East Java Province, Indonesia. J. Trop. Med. 2020, 2018, 8127093. [CrossRef]

43. Amir, M.; Hussain, A.; Asif, M.; Ahmed, S.; Alam, H.; Moga, M.A.; Cocuz, M.E.; Marceanu, L.; Blidaru, A. Full-Length Genome and Partial Viral Genes Phylogenetic and Geographical Analysis of Dengue Serotype 3 Isolates. Microorganisms $2021,9,323$. [CrossRef] [PubMed] 